\section{Effect of Mucus on the Swimming-Rate of Sperms}

IT has boen shown that bull sperm swim more slowly in mucus from the cow's uterine cervix than they do in saline ${ }^{1}$. This slowing-down might be an effect of hydroxyl ions, since the $p H$ of mucus is rather high ${ }^{2}$, and it has been claimed that media above $p \mathrm{H} 8.3$ are very harmful to sporm ${ }^{3}$. Alternatively, it might be due to the presence of inhibitory substances in solution, or to the visco-elasticity imparted to the secretion by very large flexible molecules.

Our present experiments indicate that probably it is the last of these factors which is the most important.

We have carried out experiments on the effects of varying the $p H$ of simple saline/phosphate mixtures on the swimming-rate and survival of bull sperm. In the majority of individual experiments, and in the mean for all experiments pooled, it appeared that initially both the swimming-rate and percentage of motile sperm were higher at $p H$ 8.5 than at 7.5 and 6.5 or in unbuffered saline. Although this advantage was transitory, its existence precludes explanation of the slowing down of sperm by mucus as a simple effect of $p \mathrm{H}$.

Estrous mucus may be readily separated into a gel and a supernatant by centrifugation at $44,000 \mathrm{~g}$ for $1 \frac{1}{2} \mathrm{~h}$; the viscous supernatant yields a further precipitate after $4 \mathrm{~h}$, at $103,000 \mathrm{~g}^{4}$. The final supernatant is essentially non-viscous, but contains the soluble material, mainly protein and low molecular weight substances, present in the native secretion". Tho behaviour of spermatozoa in this supernatant was examined.

The $p \mathrm{H}$, after addition of a suitable amount of semen, was in the range 7.4-7.7. Supernatants from two separate samples of mucus were tested. The first of these was used as diluent for three samples of semen, and the sperm in each case were found to swim at about four-fifths the saline rate at the same cell concentration, as compared with about two-fifths the saline rate in the whole mucus. In the second experiment two samples of semen from different bulls were made up each at two dilutions, 20,000 and 5,000 sperm per $\mu l .$, in saline and mucus supernato. Rates were measured at the boginning and also at the end of $1 \mathrm{~h}$, and at the end of $3 \mathrm{~h}$. At the begin. ning of the experiment the rate was considerably higher in mucus supernate than in saline at both dilutions, about 20 per cent higher at 20,000 and 30 60 per cent higher at 5,000. This difference persisted at the end of an hour, but at the end of $3 \mathrm{~h}$ the rates in mucus supernate were similar to those in saline.

Finally the supernatant from a large pool of a number of separate samples of mucus was tested. The rates of sperm from five samples of semen from five different bulls were higher in this material than in saline, in one caso nearly twice as high. Although the difference diminished at the end of an hour the rates in mucus supernate were all still higher than those in saline.

It appears, therefore, that neither the high $p \mathbf{H}$ nor the presence of soluble-inhibiting substances can be considerod as an explanation of the slowing down of sperm by mucus. The gel which may be spun out of the native mucus contains, after repeated washing with saline, very little of the soluble substances in the origina] secretion, but after appropriate dilution with saline retains the rheological properties of the mucus. It appears to be a complex of sialomucopolysaccharide and protein $^{5}$. In this material the spermatozoa behave as they do in native mucus, both as regards their velocity ${ }^{1}$ and their orientation along strain lines ${ }^{6}$.

This work forms part of a programme of research supported by the Population Council Inc., The Rockefeller Institute, New York.

\section{TAMPION \\ R. A. GrbBons}

National Institute for Research in Dairying, Shinfield,

Reading.

1 Tampion, D., and Gibbons, R. A., Nature, 194, 695 (1962).

${ }^{2}$ Lardy, H. A., Pounden, W. D., and Phillips, P. H., Proc. Soc. Exp, Bool., N.Y., 44, 517 (1940).

van Duijn, C., jun., and Rikmenspoel, R., J. Agric. Sci., 54, 300 (1960)

'Gibbons, R. A., Biochem. J., 78, 209 (1959).

Gibbons, R. A., and Roberts, G. P., Proc. N.Y. Acad. Sci. (in the press).

'Tampion, D., and Gibbons, R. A., Nature, 194, 381 (1962).

\section{Abnormal Crystals in Amoeba lescherae}

For the elucidation of a problem that I was investigating, it was necessary for me to have the developmental stages of Amoeba lescherae. A quick method of obtaining these is to use material from a culture grown under natural conditions ${ }^{1}$ containing a large supply of mature amoebæ. It will be remembered that in such a culture, or in a catch procured from a natural source in summer, there will be found ${ }^{1}$ : (1) mature and ageing adults; (2) encysted developmental stages of every variety; (3) spores; (4) possibly actively moving developmental stages. In the mature individuals thero will be spores and differentiating spores. A sudden change in the $p H$ (ref. 2) brought about by any agent not detrimental to the culture will kill off all the adults and so release any spores present in those adults. A study of the phenomenon is best made in Petri dishes $(4$ in. $\times 1$ in.). After the lapse of a varying period of time (a week, more or less) subsequent to the changing of the $p \mathrm{H}$, a search with the No. 7 objective water immersion lens will reveal large numbers of hatching spores, and creeping amoebulæ of various ages. But all these have a tendency to encyst, after a feed, especially at night, so the best time to search the Petri dish for active specimons is towards noon and early afternoon.

I chose for my source of material a set of three amoeba cultures grown in shallow Petri dishes (7 in. $\times 1$ in.) which, with a hand lens, were soen to be luxurious. Because of what $I$ found when I examined the adults under higher magnification, it will be necessary to state the history of these Petri dish cultures. The top culture was started on February 12,1961 , in the usual way by putting adult amoebæ plus culture fluid into Glasgow tap water containing boiled wheat grains. Food was renewed on June 12, 1961. On September 1, 1961, the culture was passed through a butter muslin net sieve to remove old grains and mattod mould growth. The filtrate was so luxurious in adults that it was divided into three sub-cultures.

In January 1962 the phenornona to be described wore discovered. I began by pipetting material from the top dish of the threo stacked culturos for exam. 